\title{
EDITORIAL
}

\section{EL COMPROMISO COLECTIVO DE LOS HOMBRES HOMOSEXUALES EN LA LUCHA CONTRA EL SIDA}

\author{
Anna Rodés Monegal \\ CEESCAT. Hospital Universitari Germans Trias i Pujol. Barcelona.
}

En España, la evolución de la epidemia del sida, así como la progresiva introducción de intervenciones preventivas que tienen como objetivo la modificación de los comportamientos de riesgo de infección por el HIV, justifican la necesidad de profundizar en el conocimiento de la evolución local de esta epidemia, mediante la creación y monitorización de indicadores de prevalencia del HIV y de los comportamientos epidemiológicamente asociados a su transmisión.

La construcción y el análisis de estos indicadores, así como el estudio de sus variaciones en el tiempo, permiten realizar un diagnóstico conductual de situación, imprescindible para el establecimiento de objetivos educativos específicos en los que fundamentar el diseño de las intervenciones preventivas. Estos indicadores también permiten iniciar y evaluar la estrategia global adoptada de prevención del sida, así como incorporar elementos técnicos en el proceso de toma de decisiones para la ejecución de políticas sanitarias, relacionadas con la planificación y programación de las actuaciones de salud pública en relación a la infección por HIV.

Aunque en la mayoría de las Comunidades Autónomas (CCAA) del estado español

Correspondencia:

Anna Rodés Monegal

CEESCAT

Hospital Universitari Germans Trias i Pujol.

Ctra de Canyet, $s / n$.

08916 Badalona. España

Correo electrónico: arodes@ceescat.hugtip.scs.es la principal vía de transmisión del HIV ha sido la parenteral, la transmisión sexual del HIV entre hombres homosexuales representa, en el conjunto de la epidemia, un relativamente alto porcentaje de casos de sida (14\%), constituyendo en algunas CCAA, como Canarias, la vía principal de contagio (36\% del total de casos) ${ }^{1}$. Sin embrago, a pesar de la importancia epidemiológica de la transmisión del HIV entre los hombres homosexuales, los estudios realizados hasta el momento en España sobre la prevalencia del HIV y de los comportamientos relacionados con su transmisión en este colectivo han sido escasos y, en gencral, limitados a cstudios de series clínicas de hombres homosexuales que acuden a los servicios sanitarios (centros de diagnóstico y tratamiento de cnfermedades de transmisión sexual o centros hospitalarios). En la mayor parte de países desarrollados, la importancia de la infección por HIV como problema de salud pública, de especial relevancia en el colectivo de hombres homosexuales, ha tenido como resultado la articulación de distintos esfuerzos preventivos desarrollados por organizaciones comunitarias de hombres homosexuales y por las instituciones de salud pública. Estos esfuerzos preventivos de la comunidad homosexual han dado resultados en la mayor parte de estos países (Estados Unidos, Canadá, Australia, Reino Unido, Suiza etcétera), habiéndose observado la mayor adopción de comportamientos sexuales más seguros en este grupo poblacional en comparación con otros grupos de población. 
Los estudios descriptivos de los comportamientos de los hombres que forman parte de la comunidad homosexual son aproximaciones útiles para evaluar y monitorizar el nivel de prevención de la infección por el HIV en dicha población.

La existencia de una asociación estructurada de hombres homosexuales no sólo es esencial para la organización de las actividades de prevención del HIV en su comunidad, sino que también lo es para evaluarlas y monitorizarlas, buena muestra de ello es el estudio presentado en este número de la Revista Española de Salud Pública sobre una muestra de hombres homosexuales de la Comunidad de Madrid ${ }^{2}$, que constituye la segunda valoración realizada en España de los comportamientos relacionados con la infección por HIV entre hombres homosexuales seleccionados fuera del sistema sanitario.

En general, los resultados de este estudio, y de otros similares realizados en la Comunidad Europea (CE), muestran cómo un número importante de homosexuales valoran el riesgo de infección por HIV y adoptan prácticas sexuales más seguras. Sin embargo, a diferencia de los estudios realizados en la CE, éste también muestra que la prevalencia de infección en este colectivo es extremadamente alta, reflejando el gran impacto que ha tenido en nuestro país la epidemia del sida.

Las diferencias en las prácticas sexuales, según las relaciones sean con una pareja estable u ocasional, muestran la complejidad del comportamiento sexual en este colectivo y cómo los comportamientos de riesgo están fuertemente influenciados por la naturaleza de la relación establecida con la pareja sexual $^{3.4}$. Así, se observa cómo en las relaciones sexuales con las parejas ocasionales, los hombres homosexuales practican menos la penetración anal y cuando la practican con este tipo de pareja, el nivel de protección que adoptan es relativamente alto. Sin embargo, es posible que los hombres homosexuales con pareja estable subestimen su grado de exposición al HIV ya que, a pesar de obser- varse una prevalencia de infección por HIV muy alta, una proporción importante de homosexuales no utiliza siempre preservativos cuando practica la penetración anal con su pareja estable. El abandono del uso del preservativo entre los miembros de la pareja estable puede que permita un mejor desarrollo de la sexualidad pero no está exento de riesgos, ya que una parte importante de las parejas estables puede desconocer su estado serológico respecto al VIH. Además, las relaciones estables a menudo no son exclusivas, debiéndose alcanzar acuerdos con la pareja estable respecto a las precauciones que deben tomarse en las relaciones sexuales fuera de la pareja. Todo ello muestra la necesidad de diversificar las intervenciones preventivas en función del tipo de relación con la pareja masculina (estable u ocasional).

En el contexto de la relación estable, la estrategia de dejar de utilizar el preservativo cuando se practica la penetración anal sólo puede ser adecuada en tres situaciones: delante de un resultado negativo del test del HIV de los dos miembros de la pareja después de que ambos hayan utilizado siempre el preservativo durante los 3 meses anteriores a la realización del test. En el caso de que los dos miembros de la pareja estable sean HIV negativos y acuerden fidelidad mutua o utilizar siempre el preservativo cuando practiquen la penetración anal con parejas ocasionales. Por último, en caso de tener relaciones sexuales de penetración anal no protegida con una pareja ocasional, estar de acuerdo en informar inmediatamente a la pareja estable y utilizar de nuevo el preservativo con ella. Por todo ello, mejorar la comunicación con la pareja establc cs un objetivo conductual especialmente relevante en la prevención de la infección por VIH. Aunque la fidelidad sea deseable por los dos miembros de la pareja estable, es recomendable recordar la importancia de alcanzar acuerdos claros con respecto a la prevención del HIV desde el inicio de la relación. Tanto en las parejas estables exclusivas (o cerradas) como en 
las no exclusivas (o abiertas), es también importante mejorar su habilidad en el establecimiento de reglas sobre cómo comunicar a la pareja la existencia de relaciones sexuales exteriores a la relación estable, así como los posibles olvidos o accidentes en la utilización de preservativos que puedan ocurrir durante estas relaciones.

Los accidentes en el manejo de los preservativos son muy frecuentes y relacionados no sólo con la frecuencia de la actividad sexual, sino también con una correcta utilización de los mismos, debiéndose realizar mayores esfuerzos para mejorar sus habilidades y el uso y acceso a lubricantes y preservativos adecuados para la práctica de la penetración anal.

La proporción de hombres que se han realizado la prueba serológica de detección de anticuerpos anti-HIV es alta, lo que sugiere que, en nuestro medio, el acceso a la prueba de detección de anticuerpos anti-HIV y el clima social existente favorecen la realización voluntaria de esta prueba.

Globalmente, los resultados muestran que la práctica del sexo seguro se está extendiendo ampliamente en la comunidad homosexual. Sin embargo, la alta prevalencia de infección por el HIV observada indica que el riesgo de transmisión del HIV en la comunidad homosexual continúa siendo importante. Así mismo, estos resultados deben ser interpretados con precaución. Distintos problemas relacionados con la selección de la muestra de hombres estudiada, la definición de «homosexualidad», «bisexualidad», «contacto sexual», etcétera, así como la validez y fiabilidad del cuestionario utilizado, deben ser tenidos en cuenta. Además, la naturaleza de la información recogida, en tanto que retrospectiva, autonotificada y relacionada con comportamientos íntimos de la persona, no permite descartar la existencia de sesgos de memoria y de subnotificación de las prácticas sexuales asociadas a mayor riesgo de infección por el HIV, aunque diversos estudios han establecido la fiabilidad de la información retrospectiva y autonotificada de los comportamientos sexuales de esta población. Por todo ello, estos resultados solo pueden ser valorados en el contexto de una asociación comunitaria de hombres homosexuales de la ciudad de Madrid. Fuera de este contexto, entre hombres homosexuales que por distintas razones no pertenecen a una organización, hombres con nivel educativo inferior, hombres que intercambian dinero por relaciones sexuales con otros hombres, la situación conductual puede variar, aunque probablemente la prevalencia de infección siga siendo tan elevada.

Los hombres homosexuales continúan siendo, por tanto, una población prioritaria en la prevención de la infección por HIV en nuestro país. Los esfuerzos dirigidos a los hombres homosexuales de todas las edades, que tengan como objetivo la promoción de comportamientos sexuales seguros, deben ser intensificados y mantenidos.

Finalmente, la introducción en el año 1996 de las nuevas terapias antivirales combinadas y en 1997 de las nuevas posibilidades de profilaxis post-exposición sexual al HIV pueden haber modificado las circunstancias en las que ocurre la infección por HIV. El aumento de la supervivencia y la mejora de la calidad de vida de las personas infectadas hacen esperar que la prevalencia sea alta, al ser ésta un parámetro afectado por la duración de la infección. No obstante, estos avances terapéuticos pueden tener efectos negativos al hacer temer que puedan incitar a determinadas personas a protegerse menos que antes. Por ello, el análisis de las características socio-demográficas y conductuales de este colectivo y su monitorización en el tiempo, junto con el análisis de la información proporcionada por los sistemas de vigilancia epidemiológica del HIV establecidos en nuestro medio, permitirán ampliar el conocimiento local sobre la evolución de la epidemia y mejorar la organización de los servicios sanitarios y las actividades de salud pública que deben llevarse a cabo en relación al sida y la infección por HIV entre hombres homosexuales. 


\section{BIBLIOGRAFÍA}

1. Ministerio de Sanidad y Consumo. Secretaria del Plan Nacional del Sida. Centro Nacional de Epidemiología. Vigilancia del Sida en España. Situación al 30 de junio de 1999. Registro Nacional del Sida. Madrid: Ministerio de Sanidad y Consumo; 1999.

2. Conductas sexuales de riesgo y prevalencia de infección por HIV en hombres con prácticas ho- mosexuales y bisexuales en la comunidad de Madrid. Rev Esp Salud Pública 2000; 74: 25-32.

3. Fitzpatrick R, Boulton M, Hart G, Dawson J, McLean J. High risk sexual behaviour and condom use in a sample of homosexual and bisexual men. Health Trends 1989;21: 76-79.

4. McKusick L, Horstman W, Coates T. Aids and sexual behavior reported by gay men in San Francisco. Am J Public Health 1985; 75:493496. 